ఠ

\title{
circSMAD2 inhibits the epithelial-mesenchymal transition by targeting miR-629 in hepatocellular carcinoma
}

This article was published in the following Dove Press journal:

OncoTargets and Therapy

\section{Xianwei Zhang' \\ Ping Luo' \\ Wei Jing' \\ $\mathrm{Hu}$ Zhou $^{2}$ \\ Chunzi Liang' \\ Jiancheng Tu'}

'Department of Clinical Laboratory Medicine, Center for Gene Diagnosis, Zhongnan Hospital of Wuhan University, Wuhan, People's Republic of China; ${ }^{2}$ Department of Blood Transfusion, Tongji Hospital, Tongji Medical College, Huazhong University of Science and Technology, Wuhan, People's Republic of China
Correspondence: jiancheng Tu Department \& Program of Clinical Laboratory Medicine, Center for Gene Diagnosis, Zhongnan Hospital of Wuhan University, 169 Donghu Road, Wuhan 43007I, People's Republic of China

Tel +862767812989

Fax +862767813233

Email jianchengtu@whu.edu.cn
Background: Circular RNAs (circRNAs) are a class of widely distributed non-coding RNAs, which drew little attention for decades. Recent studies show that circRNAs are involved in cancer progression.

Methods: The circSMAD2 expression in HCC and adjacent non-tumor tissues was measured by quantitative real-time polymerase chain reaction, and the biological function of circSMAD2 was explored by proliferation, apoptosis, migration, invasion, and Western blot assays. Next, the dual-luciferase reporter assay was performed to identify the target miRNA of circSMAD2. Finally, circSMAD2 and its target miRNA were co-transfected in HCC cells to investigate their relationship to HCC progression.

Results: In this study, we found that circRNA SMAD2 (circSMAD2) expression was downregulated in hepatocellular carcinoma (HCC) tissues $(P=0.014)$ compared to the adjacent non-tumor tissues and markedly associated with the differentiation degree of the HCC tissues $(P<0.001)$. The in vitro experiments showed that overexpressed circSMAD2 inhibited the migration, invasion, and epithelial-mesenchymal transition (EMT) in HCC cells. Bioinformatics predicted that miR629 is a potential target of circSMAD2, and the dual-luciferase reporter assay verified that miR-629 directly bound circSMAD2. In addition, we found that overexpression of circSMAD2 suppressed the expression of miR-629 in HCC cells, whereas knockdown of circSMAD2 upregulated the expression of miR-629. Furthermore, co-transfection of miR-629 mimics with circSMAD2 reversed the circSMAD2 effects of inhibiting the migration, invasion, and EMT of HCC cells. Conclusion: Altogether, our data support that circSMAD2 inhibits the migration, invasion, and EMT of HCC cells by targeting miR-629.

Keywords: circular RNA, circSMAD2, hepatocellular carcinoma, epithelial-mesenchymal transition, microRNA-629

\section{Introduction}

Hepatocellular carcinoma (HCC) is the third leading cause of cancer-related deaths worldwide. ${ }^{1,2}$ Surgical resection during early stages is a curative treatment that improves the 5-year HCC survival rate. ${ }^{3}$ However, HCC patients are frequently diagnosed at an advanced stage, which indicates a poor prognosis. ${ }^{4}$ Therefore, a better understanding of the HCC progression mechanism and the identification of novel diagnostic markers and therapeutic targets for HCC are urgent.

Circular RNAs (circRNAs) are a large class of endogenous non-coding RNAs (ncRNAs) that covalently link the $5^{\prime}$ and $3^{\prime}$ ends together and form circular loops. ${ }^{5}$ CircRNAs are abundant, conserved, and stable and have tissue-specific expression in mammalian cells..$^{5-8}$ CircRNAs play important roles in cellular activities, such as modulating the expression of parental genes ${ }^{9}$ and functioning as microRNA (miRNA) (c)
hereby accept the Terms. Non-commercial uses of the work are permitted without any further permission from Dove Medical Press Limited, provided the work is properly attributed. For permission hereby accept the Terms. Non-commercial uses of the work are permitted without any further permission from Dove Med commercial use of this work, please see paragraphs 4.2 and 5 of our Terms (https://www.dovepress.com/terms.php).
for 
sponges. ${ }^{10}$ In addition, circRNAs are involved in different types of cancers, including HCC. ${ }^{11-15}$ For example, circMTO1 is significantly downregulated in HCC tissues, and the deceased amount of circMTO1 is a prognosis predictor for poor survival of patients. ${ }^{16}$

The circRNA SMAD2 (circSMAD2; circBase ID: Hsa_ circ_0000847) is encoded by the SMAD2 gene and located at chr18:45391429-45423180. CircSMAD2 is an exon-intron circRNA (EIcircRNA) that is circularized with both exons and introns simultaneously. Several EIcircRNAs can upregulate the expression of their parental genes. ${ }^{9,17}$ SMAD2, the parental gene of circSMAD2, plays a key role in the epithelial-mesenchymal transition (EMT), which promotes the stationary epithelium-derived tumor cells to become migratory and invasive and plays a vital role in the progression of HCC. ${ }^{18}$ In addition, the expression of circSMAD2 is upregulated during TGF- $\beta$-induced EMT. ${ }^{19}$ Based on the abovementioned information, we investigated the relationship between circSMAD2 and HCC in this study.

\section{Materials and methods}

\section{Patient data and specimen collection}

A total of 86 pairs of $\mathrm{HCC}$ and adjacent non-tumor tissues were obtained from HCC patients (82 males and four females, mean age $54 \pm 10$ years) who underwent surgery without preoperative chemotherapy or radiotherapy at the Zhongnan Hospital of Wuhan University from 2011 to 2015. All patients were selected based on their pathology reports. Tumor staging (stages I, II, III, and IV) was defined according to the seventh edition of the AJCC Cancer Staging Manual. Tumor specimens and paired adjacent non-tumor tissues were stored at $-80^{\circ} \mathrm{C}$ in RNAlater ${ }^{\circledR}$ RNA Stabilization Solution (Thermo Fisher Scientific, Waltham, MA, USA).

\section{Ethical approval}

The study conforms with the Code of Ethics of the World Medical Association (Declaration of Helsinki) printed in the British Medical Journal (18 July 1964). In addition, the study was approved by the ethics committee of Zhongnan Hospital of Wuhan University, and all patients signed the written informed consent.

\section{RNA extraction and reverse transcription}

Total RNA content of tissues and plasma was extracted using the TRIzol reagent (Thermo Fisher Scientific) according to the manufacturer's instructions. The concentration and purity of the obtained RNA samples were quantified using the NanoDrop ND2000 (Thermo Fisher Scientific). The RNA samples were reverse transcribed to cDNA using the PrimeScript ${ }^{\mathrm{TM}}$ RT Reagent Kit with gDNA Eraser (Takara, Dalian, China) according to the manufacturer's instructions.

\section{Quantitative real-time polymerase chain} reaction ( $q R T-P C R$ )

qRT-PCR was performed with SYBR $^{\circledR}$ Premix Ex Taq ${ }^{\mathrm{TM}}$ II (Takara) on the Bio-Rad CFX96 (Bio-Rad Laboratories Inc., Hercules, CA, USA) according to the manufacturer's instructions. The related primer sequences are listed in Table S1. The reactions began with an initial denaturation at $95^{\circ} \mathrm{C}$ for $5 \mathrm{~min}$, followed by denaturation at $95^{\circ} \mathrm{C}$ for $30 \mathrm{~s}$, annealing at $63.3^{\circ} \mathrm{C}$ for $30 \mathrm{~s}$, and extension at $72^{\circ} \mathrm{C}$ for $30 \mathrm{~s}$. The denaturation, annealing, and extension steps were repeated for 40 cycles. The glyceraldehyde 3-phosphate dehydrogenase (GAPDH) and U6 genes were used as internal controls for circRNAs and miRNAs, respectively.

\section{Plasmid construction}

We used the pcDNA3.1(+) circRNA mini vector (Addgene plasmid 60648), which is a circRNA-forming plasmid obtained from Liang and Wilusz ${ }^{20}$ to subclone the circSMAD2 sequence for the transfection studies. In addition, psiCHECK2 (Promega Corporation, Fitchburg, WI, USA) was used in the dual-luciferase reporter assay. The circSMAD2cDNA was amplified using PCR with specific primers. The obtained PCR fragments were subcloned into the EcoRV and SacII sites of the pcDNA3.1(+) circRNA mini vectors. The wild-type circSMAD2 (circSMAD2-wt) and the mutanttype circSMAD2 (circSMAD2-mut) were subcloned into the XhoI and NotI sites of the psiCHECK2 vectors, respectively. Afterward, the reconstructed plasmids were validated using DNA sequencing. Finally, more copies of the reconstructed plasmids were produced and purified using the Mini Plasmid Preparation Kit (Axygen, Hangzhou, China) according to the manufacturer's instructions. In addition, small interfering RNA of circSMAD2 (si-circSMAD2; GenePharma, Suzhou, China) was synthesized to knock down the expression of circSMAD2, and miR-629 mimics (GenePharma) was synthesized to overexpress miR-629 in vitro (Table S2).

\section{Cell culture and plasmid transfection}

The HCC cell lines HepG2, HCCLM9, HCCLM3, and MHCC97L as well as the immortalized human hepatic cell line L02 were obtained from the Cell Bank of Type Culture Collection of Chinese Academy of Sciences (Shanghai, China). Cells were cultured in DMEM (Thermo Fisher Scientific) with $10 \%$ fetal bovine serum (FBS; Thermo Fisher Scientific) in a humidified incubator at $37^{\circ} \mathrm{C}$ with $5 \% \mathrm{CO}_{2}$. A six-well plate was seeded with $5 \times 10^{5}$ cells and incubated for $24 \mathrm{~h}$. The cells were then transfected with the related genes using Lipofectamine ${ }^{\mathrm{TM}} 2000$ (Thermo Fisher Scientific) according to the manufacturer's instructions. 


\section{Cell proliferation assay}

Cell proliferation assays were conducted using the Cell Counting Kit-8 (CCK-8) (Dojindo, Kumamoto, Japan) according to the manufacturer's instructions. In brief, transfected cells were seeded into 96 -well plates (2,000 cells/well) and cultured for 0 , 24,48 , and $96 \mathrm{~h}$. Then, $10 \mu \mathrm{L}$ of CCK-8 solution was added to each well, and the plates were incubated at $37^{\circ} \mathrm{C}$ for an additional $2 \mathrm{~h}$. Finally, the solution was measured at $450 \mathrm{~nm}$ on the spectrophotometer (EnSpire; PerkinElmer Inc., Waltham, MA, USA).

\section{Flow cytometric analysis}

Transfected HepG2 cells were harvested after transfection for $24 \mathrm{~h}$ and stained using an Annexin V-FITC/PI Apoptosis Detection Kit (Beyotime, Shanghai, China) according to the manufacturer's instructions. The cells were then analyzed using a Cytomics ${ }^{\mathrm{TM}}$ FC 500 flow cytometer (Beckman Coulter, Brea, CA, USA).

\section{Cell migration and invasion assays}

Transfected HepG2 cells were harvested after transfection for $24 \mathrm{~h}$ and seeded into the upper chambers $(20,000$ cells/chamber) of Transwell assay plates (Corning Incorporated, Corning, NY, USA) with $200 \mu \mathrm{L}$ of serum-free DMEM to quantify cell migration. Similarly, transfected HepG2 cells were seeded into the upper chambers (40,000 cells/chamber) of Transwell plates with Matrigel-coated membranes (BD, Franklin Lakes, NJ, USA) in $200 \mu \mathrm{L}$ serum-free DMEM to quantify cell invasion. The lower chambers were filled with DMEM containing $10 \% \mathrm{FBS}$. After an incubation period of $24 \mathrm{~h}$, the medium was removed and cells were fixed with methanol for $20 \mathrm{~min}$. The cells were stained with crystal violet for $20 \mathrm{~min}$, air-dried, and photographed using a digital microscope. The number of cells was calculated from five random fields in each chamber.

\section{Western blot}

Cells were lysed for extraction of total protein. After measurement of protein concentration, the protein was separated using $10 \%$ sodium dodecyl sulfate polyacrylamide gel electrophoresis (SDS-PAGE) and transferred onto polyvinylidene difluoride (PVDF) membranes (EMD Millipore, Billerica, MA, USA). After blocking with 5\% non-fat milk, the membranes were incubated with primary antibodies: anti-SMAD2 (Abcam, Cambridge, MA, USA), anti-GAPDH (Abcam), anti-E-cadherin (Cell Signaling Technology, Danvers, MA, USA), anti-Ncadherin (Cell Signaling Technology), anti-Snail (Cell Signaling Technology), and anti-Vimentin (Cell Signaling Technology). Then the membranes were incubated with horseradish peroxidase-conjugated secondary antibodies. Immunoblot signals were visualized using ECL Plus Kit (GE Healthcare, Fairfield, CT, USA) according to the manufacturer's instructions.

\section{Dual-luciferase reporter assay}

HepG2 cells were co-transfected with $4 \mu \mathrm{g}$ psiCHECK-2circSMAD2-wt or orpsiCHECK-2-circSMAD2-mut plasmids and $20 \mathrm{nmol} / \mathrm{L}$ miR-629 mimics or miRNA negative control (miR-NC) using Lipofectamine ${ }^{\mathrm{TM}} 2000$ (Thermo Fisher Scientific) according to the manufacturer's instructions. After $48 \mathrm{~h}$ of incubation, the Firefly and Renilla luciferase activities were measured using a dual-luciferase reporter assay kit (Promega Corporation) according to the manufacturer's instructions.

\section{Statistical analyses}

All statistical analyses were carried out using SPSS version 21.0 (IBM Corporation, Armonk, NY, USA) and GraphPad Prism 5.0 (GraphPad Software, Inc., La Jolla, CA, USA). The numerical data are presented as mean $(M) \pm$ standard error of the mean (SEM) of at least three determinations. Results were considered as statistically significant at $P<0.05$. The normality of distribution for each data set was tested using the ShapiroWilk test. Normally distributed data sets were analyzed using the Student's $t$-tests, while non-normally distributed data sets were analyzed using Kruskal-Wallis variance analyses.

\section{Results}

\section{CircSMAD2 was downregulated in} HCC tissues and cell lines and markedly associated with the differentiation degree of HCC tissues

To elucidate the circSMAD2 profile in HCC, we performed qRT-PCR in 86 pairs of HCC and adjacent non-tumor tissues. CircSMAD2 was downregulated in HCC tissues compared with non-tumor tissues $(P=0.014$; Figure 1A). Comparison of different clinical features (Table 1) indicated that the expression of circSMAD2 was significantly lower in the poorly differentiated HCC tissues than in the high/moderate ones $(P<0.001$; Figure 1B). We further detected circSMAD2 expression in the HCC and hepatic cell lines. The qRT-PCR results showed that circSMAD2 was downregulated in the HCC cell lines HCCLM9, HCCLM3, HCC97L, and HepG2 compared with the hepatic cell line L02 (Figure 1C).

\section{Overexpression of circSMAD2 inhibited the migration, invasion, and EMT of HCC cells}

To investigate the biological function of circSMAD2, we overexpressed circSMAD2 in HepG2 cells (Figure 2A), which had the lowest circSMAD2 expression of the four 
A

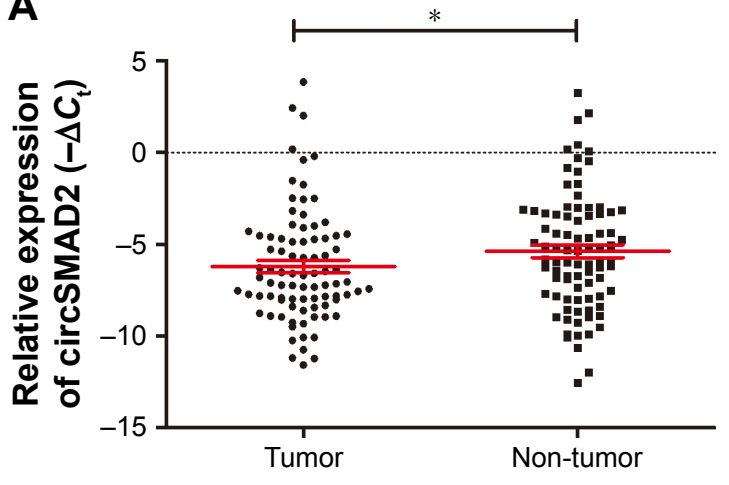

B

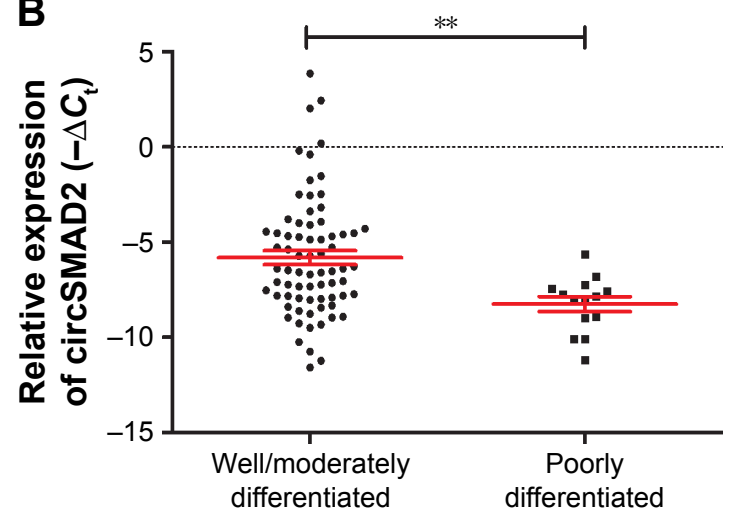

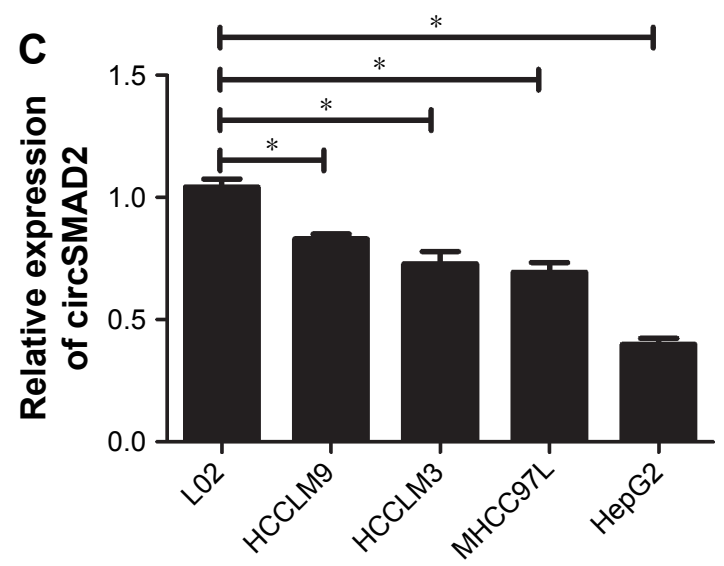

Figure I CircSMAD2 was downregulated in HCC tissues and cell lines and markedly associated with the differentiation degree of HCC tissues.

Notes: (A) CircSMAD2 was downregulated in the HCC tissues compared with the adjacent non-tumor tissues. (B) The expression of circSMAD2 was significantly lower in the poorly differentiated HCC tissues than in the high/moderate ones. (C) CircSMAD2 was downregulated in the HCC cell lines HCCLM9, HCCLM3, HCC97L, and HepG2 compared with the hepatic cell line L02. The expression levels of circSMAD2 were represented with $-\Delta C_{t}$ value in each sample. The $-\Delta C_{t}$ value was determined by subtracting the $C_{t}$ value of circRNA from the $C_{t}$ value of GAPDH. Data are presented as mean \pm SEM. $* P<0.05$ and $* * P<0.01$.

Abbreviations: circSMAD2, circRNA SMAD2; HCC, hepatocellular carcinoma; circRNA, circular RNA; GAPDH, glyceraldehyde 3-phosphate dehydrogenase; SEM, standard error of the mean.

HCC cell lines. The Transwell assay showed that overexpression of circSMAD2 inhibited the migration and invasion of HepG2 cells (Figure 2B and C), whereas the CCK-8 and flow cytometric assays showed no significant changes in the proliferation and apoptosis of these cells (Figure S1A and B). The EMT plays a key role in the tumor metastasis. Thus, we determined if circSMAD2 could regulate the EMT in HCC cells. Western blot assays showed that the epithelial cell marker E-cadherin was significantly upregulated, whereas the mesenchymal cell markers N-cadherin, Snail, and Vimentin were significantly reduced in circSMAD2-overexpressed HepG2 cells (Figure 3), suggesting that circSMAD2 inhibited the EMT process in HCC cells.

Several EIcircRNAs can regulate the expression of parental genes, and circSMAD2 is an EIcircRNA. In addition, SMAD2 protein plays a key role in the EMT process. Thus, we explored whether circSMAD2 regulated the EMT in HCC by regulating SMAD2 expression. However, the results indicated no significant change in SMAD2 expression with overexpressed or knocked down circSMAD2 (Figure S1C) HepG2 cells (Figure 4).

\section{CircSMAD2 acts as an miRNA sponge for miR-629 in HCC cells}

To explore whether circSMAD2 is serving as an miRNA sponge in $\mathrm{HCC}$, we searched for potential target miRNAs using a bioinformatics database (circRNA interactome). Among the potential candidates, we focused on miRNAs involved in the EMT. miR-629 attracted our attention (Figure 5A) because it induces tumor initiation and growth of $\mathrm{HCC}$ and promotes the EMT in several cancer cell lines..$^{21,22}$ To confirm whether miR-629 was the direct target of circSMAD2, we performed a dual-luciferase reporter assay. As shown in Figure 5B, the relative luciferase activity was significantly lower in HepG2 cells co-transfected with the psiCHECK2-circSMAD2-wt and miR-629 compared with controls, but no significant difference was detected in the HepG2 cells co-transfected with psiCHECK2-circSMAD2-mut and miR-629 compared 
Table I Correlation between circSMAD2 expression $\left(-\Delta C_{t}\right)$ and clinical features of HCC patients

\begin{tabular}{|c|c|c|c|}
\hline $\begin{array}{l}\text { Clinical } \\
\text { features }\end{array}$ & $\begin{array}{l}\text { Patient } \\
\text { number }\end{array}$ & $\begin{array}{l}\text { Relative } \\
\text { circSMAD2 } \\
\text { expression }\left(-\Delta C_{t}\right) \\
\text { mean } \pm \text { SEM }\end{array}$ & $P$-value \\
\hline Gender & & & 0.438 \\
\hline Female & 6 & $-5.27 \pm 0.8 \mid$ & \\
\hline Male & 80 & $-6.28 \pm 0.35$ & \\
\hline Age & & & 0.810 \\
\hline$<54$ & 43 & $-6.28 \pm 0.45$ & \\
\hline$\geq 54$ & 43 & $-6.13 \pm 0.48$ & \\
\hline Smoking & & & 0.338 \\
\hline Positive & 49 & $-6.48 \pm 0.52$ & \\
\hline Negative & 37 & $-5.84 \pm 0.43$ & \\
\hline Alcoholism & & & 0.276 \\
\hline Positive & 52 & $-6.40 \pm 0.36$ & \\
\hline Negative & 32 & $-5.76 \pm 0.62$ & \\
\hline Tumor size & & & 0.904 \\
\hline$<5 \mathrm{~cm}$ & 33 & $-6.15 \pm 0.43$ & \\
\hline$\geq 5 \mathrm{~cm}$ & 53 & $-6.23 \pm 0.47$ & \\
\hline Tumor focal & & & 0.865 \\
\hline Single & 60 & $-6.16 \pm 0.41$ & \\
\hline Multiple & 26 & $-6.29 \pm 0.54$ & \\
\hline TNM & & & 0.301 \\
\hline Early & 38 & $-6.59 \pm 0.47$ & \\
\hline Late & 48 & $-5.90 \pm 0.46$ & \\
\hline Differentiation** & & & $<0.001$ \\
\hline High/moderate & 72 & $-5.8 I \pm 0.37$ & \\
\hline Low & 14 & $-8.25 \pm 0.39$ & \\
\hline $\mathrm{ALT}$ & & & 0.554 \\
\hline$\geq 46$ & 49 & $-6.03 \pm 0.42$ & \\
\hline$<46$ & 37 & $-6.43 \pm 0.53$ & \\
\hline AST & & & 0.468 \\
\hline$\geq 46$ & 44 & $-5.97 \pm 0.50$ & \\
\hline$<46$ & 42 & $-6.45 \pm 0.43$ & \\
\hline AFP & & & 0.740 \\
\hline$\geq 200$ & 44 & $-6.3 I \pm 0.48$ & \\
\hline$<200$ & 42 & $-6.09 \pm 0.46$ & \\
\hline GGT & & & 0.096 \\
\hline$\geq 55$ & 38 & $-5.59 \pm 0.54$ & \\
\hline$<55$ & 48 & $-6.69 \pm 0.40$ & \\
\hline GLU & & & 0.648 \\
\hline$<6.2$ & 75 & $-6.27 \pm 0.34$ & \\
\hline$\geq 6.2$ & 11 & $-5.8 \mathrm{I} \pm \mathrm{I} .13$ & \\
\hline
\end{tabular}

Notes: Data are presented as mean \pm SEM. $* * P<0.01$.

Abbreviations: circSMAD2, circRNA SMAD2; HCC, hepatocellular carcinoma; SEM, standard error of the mean; ALT, alanine aminotransferase; AST, aspartate aminotransferase; AFP, $\alpha$-fetoprotein; GGT, $\gamma$-glutamyltransferase; GLU, glucose.

with controls, suggesting that miR-629 directly bound circSMAD2. In contrast to the expression levels of circSMAD2, miR-629 was upregulated in the HCC cell lines HCCLM9, HCCLM3, HCC97L, and HepG2 compared with the hepatic cell line L02 (Figure 5C). In addition, overexpression of circSMAD2 reduced the expression of miR-629 in HepG2 cells, whereas knockdown of circSMAD2 upregulated the expression of miR-629 (Figure 5D). Collectively, circSMAD2 might act as an miRNA sponge for miR-629 in HCC cells.

\section{miR-629 could reverse the circSMAD2 effects of inhibiting the migration, invasion, and EMT of HCC cells}

To investigate whether circSMAD2 regulates the EMT in HCC cells by targeting miR-629, the miR-629 mimics and pcDNA 3.1-circSMAD2 were co-transfected into HepG2 cells (Figure S2). The Transwell and Western blot assays showed that co-transfection with miR-629 mimics and pcDNA 3.1-circSMAD2 reversed the circSMAD2 effect of inhibiting the migration, invasion, and EMT of HCC cells compared with co-transfection with miR-NC and pcDNA 3.1-circSMAD2 (Figure 6A-C). Collectively, these data suggest that circSMAD2 might inhibit the migration, invasion, and EMT by targeting miR-629 in HCC cells.

\section{Discussion}

Because of the development of RNA sequencing technologies and bioinformatics, the functions of circRNAs were revealed, such as modulating the expression of parental genes and serving as miRNA sponges. Furthermore, recent studies show that circRNAs participate in the initiation and progression of tumors, including HCC. ${ }^{12,16,23}$

In this study, we found that circSMAD2 expression was downregulated in HCC tissues compared with the adjacent non-tumor tissues, and the expression levels of circSMAD2 were significantly lower in the poorly differentiated tissues than in the well differentiated/moderately differentiated ones. The in vitro experiments showed that circSMAD2 overexpression inhibited the migration and invasion of $\mathrm{HCC}$ cells. The EMT is the mechanism that drives a transient and reversible de-differentiation of epithelial cells to a mesenchymal-like phenotype and contributes to tumor metastasis of various cancers, including HCC. ${ }^{24}$ Thus, we explored whether circSMAD2 was involved in the EMT in HCC cells. The results showed that overexpression of circSMAD2 inhibited the EMT in HCC cells. CircSMAD2 is an EIcircRNA, and several EIcircRNAs can regulate the expression of parental genes. In addition, $S M A D 2$, the parental gene of circSMAD2, plays a key role in the EMT. Thus, we hypothesized that circSMAD2 might regulate the EMT by modulating SMAD2 expression. However, no significant change in the SMAD2 expression was found in HCC cells with overexpressed or knocked down circSMAD2, suggesting that circSMAD2 might have no influence on the SMAD2 expression. 

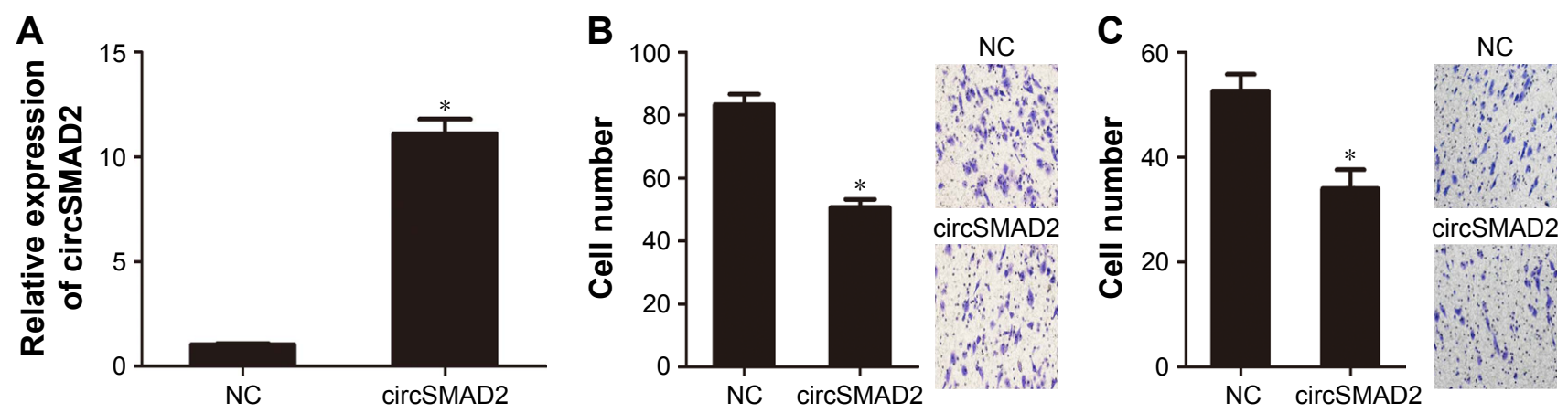

Figure 2 circSMAD2 was over-expressed in $\mathrm{HCC}$ cells and inhibited the migration and invasion of $\mathrm{HCC}$ cells.

Notes: (A) After transfection with pcDNA3.I-circSMAD2 for 24 h, the circSMAD2 was significantly upregulated in HepG2 cells. Overexpression of circSMAD2 inhibited the migration $(\mathbf{B})$ and invasion $(\mathbf{C})$ of HCC cells. Data are presented as mean $\pm \mathrm{SEM}$. $* P<0.05$.

Abbreviations: circSMAD2, circRNA SMAD2; HCC, hepatocellular carcinoma; SEM, standard error of the mean; circRNA, circular RNA; NC, negative control.

A previous study showed that circRNAs are able to serve as miRNA sponges. ${ }^{10}$ To explore the underlying mechanism of circSMAD2 in HCC, the potential miRNA targets of circSMAD2 were predicted using bioinformatics. Then, we focused our attention on miR-629, because not only it is one of the high-ranked targets but also it plays a vital role in the HCC oncogenesis. ${ }^{21}$ The subsequent dual-luciferase reporter assay indicated that miR-629 directly bound circSMAD2. In contrast to the expression levels of circSMAD2, we found that miR-629 expression was upregulated in HCC cells. In addition, circSMAD2 overexpression induced miR-629 downregulation in HCC cells, whereas knockdown of circSMAD2 upregulated the expression of miR-629. Furthermore, co-transfection of circSMAD2 and miR-629 reversed the circSMAD2 effects of inhibiting the migration, invasion, and EMT of HCC cells. Hatziapostolou et $\mathrm{al}^{21}$ revealed that miR-629 is upregulated in both HCC cell lines and tumors and plays a key role in HCC initiation and growth.
In addition, miR- 629 promotes the EMT, motility, and invasion in clear renal cell carcinoma by downregulating the expression of tripartite motif-containing 33 (TRIM33), which regulates the EMT via inhibiting the TGF- $\beta / \operatorname{Smad} 2$ signaling pathway. ${ }^{22,25-27}$ TRIM33 expression is reduced in HCC tissues, and the reduced expression of TRIM33 promotes the HCC metastasis. ${ }^{28}$ Therefore, regulating miR629 expression and its downstream functions might partly contribute to the effect of circSMAD2 on regulating the EMT of HCC cells. However, the detailed mechanism requires further investigation.

The main limitation of our study is that our experiments were done in vitro and the biological function of circSMAD2 remains to be validated in vivo. Besides, we only verified the relationship between miR-629 and circSMAD2. The potential relationships between other predicted target miRNAs and circSMAD2 remain to be further investigated in HCC.
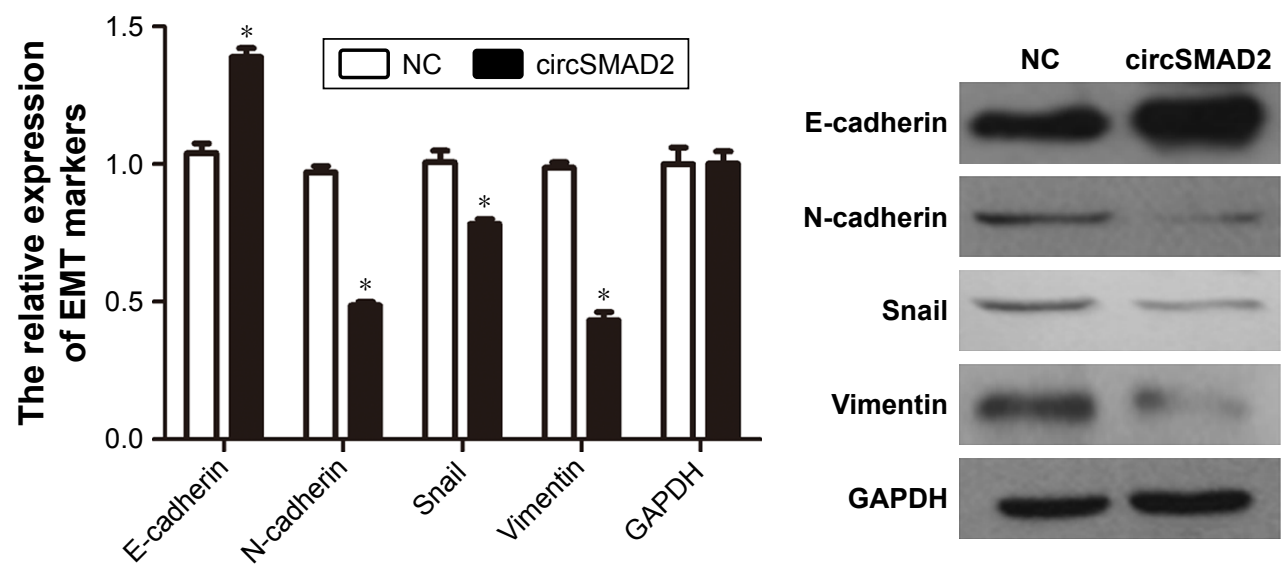

Figure 3 Overexpression of circSMAD2 upregulated the epithelial cell marker E-cadherin and downregulated the mesenchymal cell markers $\mathrm{N}$-cadherin, Snail, and Vimentin in HepG2 cells.

Notes: Data are presented as mean \pm SEM. $* P<0.05$.

Abbreviations: circSMAD2, circRNA SMAD2; SEM, standard error of the mean; circRNA, circular RNA; EMT, epithelial-mesenchymal transition; NC, negative control; GAPDH, glyceraldehyde 3-phosphate dehydrogenase. 
A

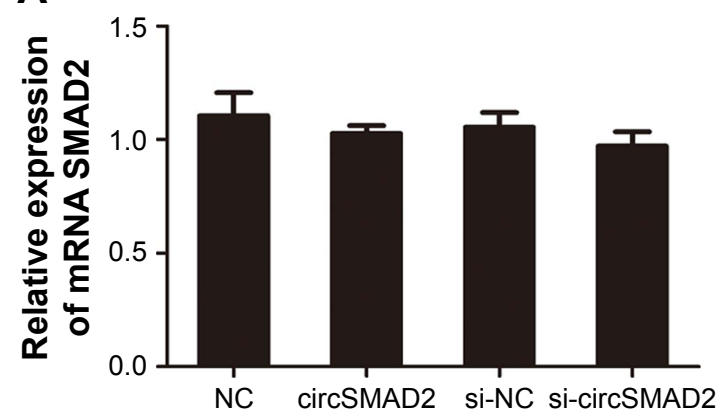

B

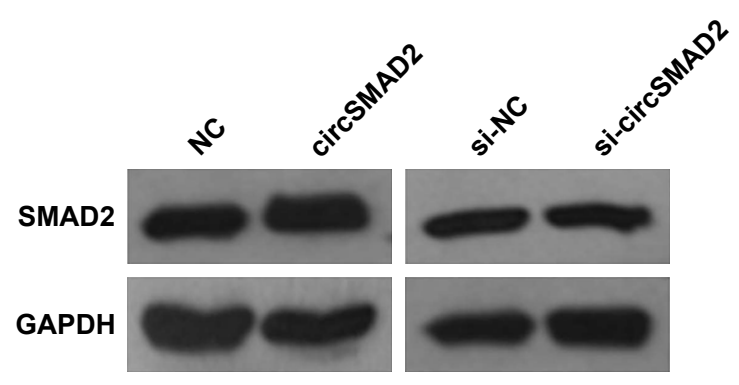

Figure 4 QRT-PCR and Western blot assays showed no significant changes in the mRNA (A) or protein levels (B) of SMAD2 in overexpressed or knocked down circSMAD2 HepG2 cells.

Note: Data are presented as mean \pm SEM.

Abbreviations: QRT-PCR, quantitative real-time polymerase chain reaction; circSMAD2, circRNA SMAD2; SEM, standard error of the mean; NC, negative control; GAPDH, glyceraldehyde 3-phosphate dehydrogenase.

\section{Conclusion}

We found that circSMAD2 was downregulated in HCC tissues and markedly associated with the differentiation degree of tumors. In vitro experiments revealed that circSMAD2 inhibited the migration, invasion, and EMT of HCC cells. Furthermore, we demonstrated that miR-629 directly binds circSMAD2 and circSMAD2 inhibits the migration, invasion, and EMT of HCC cells by suppressing miR-629 expression.

A

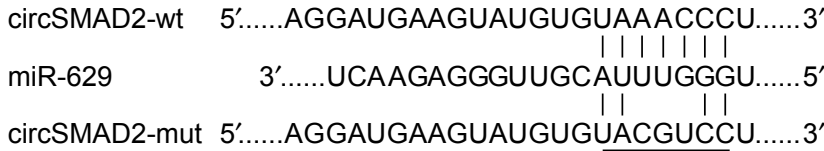
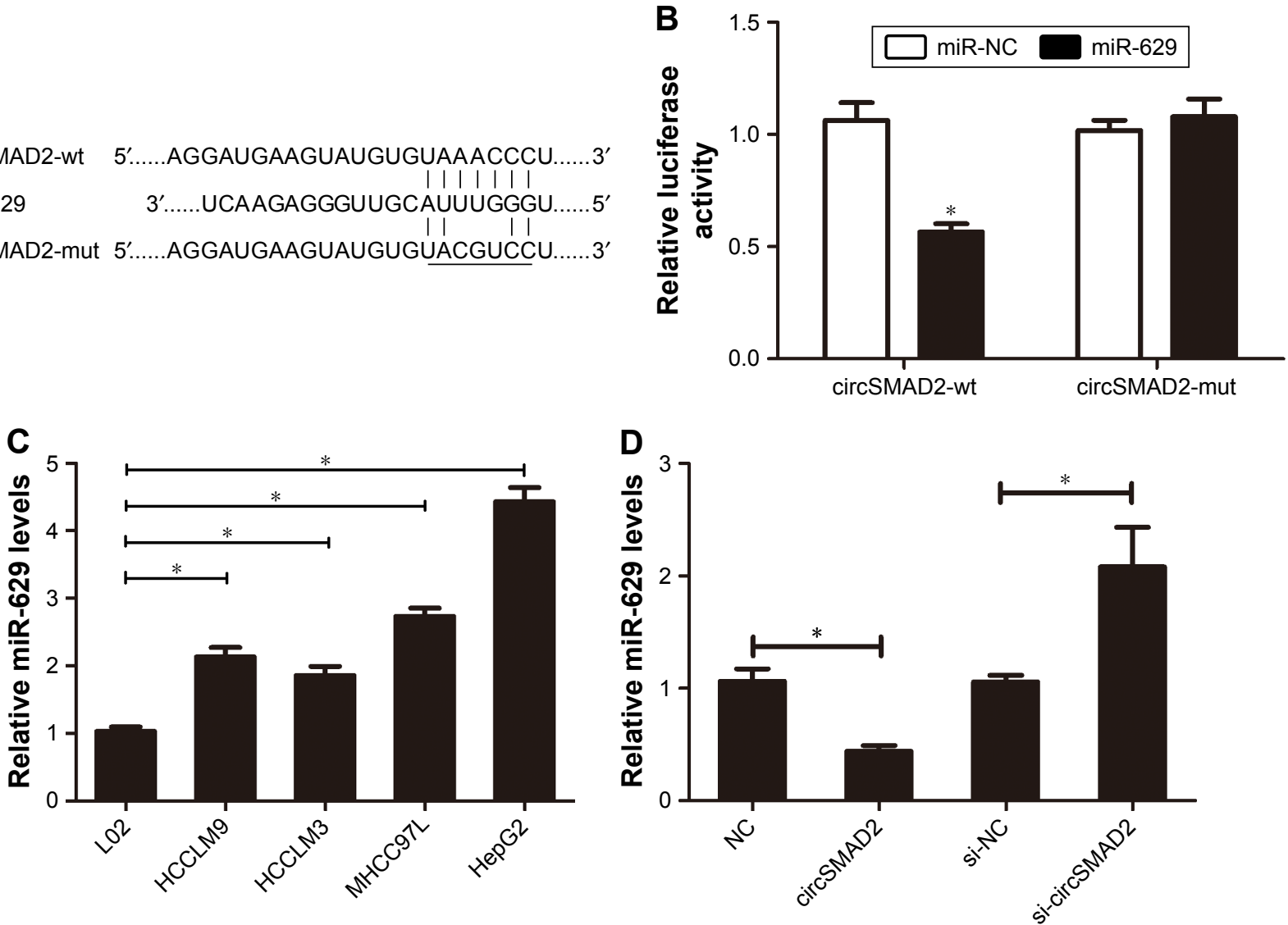

Figure 5 circSMAD2 acts as an miRNA sponge for miR-629 in HCC cells.

Notes: (A) The predicted target sites of circSMAD2 and miR-629. (B) The relative luciferase activity (Renilla/Firefly) of the reporter was significantly lower in HepG2 cells co-transfected with the psiCHECK2-circSMAD2-wt and miR-629 mimics compared with control, whereas no significant change was found in the HepG2 cells co-transfected with psiCHECK2-circSMAD2-mut and miR-629 compared with control. (C) miR-629 was upregulated in the HCC-derived cell lines HCCLM9, HCCLM3, HCC97L, and HepG2 compared with the hepatic cell line L02. (D) Overexpression of circSMAD2 reduced the expression of miR-629 in HepG2 cells, while circSMAD2 knockdown upregulated the expression level of miR-629 in HepG2 cells. Data are presented as mean \pm SEM. $* P<0.05$.

Abbreviations: circSMAD2, circRNA SMAD2; HCC, hepatocellular carcinoma; circSMAD2-wt, wild-type circSMAD2; circSMAD2-mut, mutant-type circSMAD2; SEM, standard error of the mean; circRNA, circular RNA; NC, negative control. 

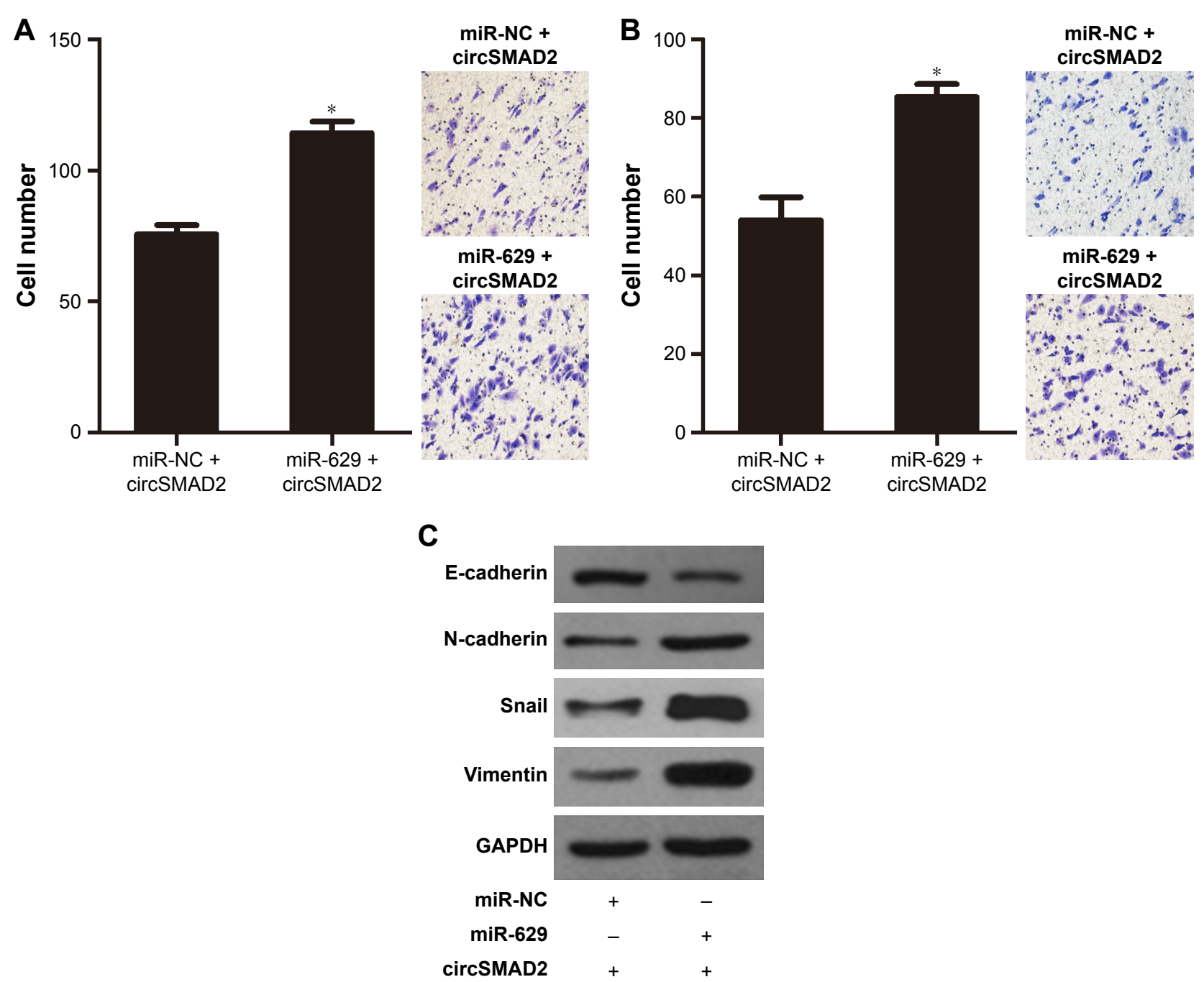

Figure 6 miR-629 reversed the effect of circSMAD2 on the migration, invasion, and EMT of HCC cells.

Notes: Co-transfection with miR-629 mimics reversed the effect of circSMAD2 on inhibiting the migration (A), invasion (B), and EMT (C) of HCC cells. Data are presented as mean \pm SEM. $* P<0.05$.

Abbreviations: circSMAD2, circRNA SMAD2; EMT, epithelial-mesenchymal transition; HCC, hepatocellular carcinoma; SEM, standard error of the mean; circRNA, circular RNA; NC, negative control; GAPDH, glyceraldehyde 3-phosphate dehydrogenase.

\section{Acknowledgment}

This study was supported by the National Basic Research Program of China (973 Program; 2012CB720605).

\section{Disclosure}

The authors report no conflicts of interest in this work.

\section{References}

1. Jemal A, Bray F, Center MM, Ferlay J, Ward E, Forman D. Global cancer statistics. CA Cancer J Clin. 2011;61(2):69-90.

2. El-Serag HB. Hepatocellular carcinoma. $N$ Engl J Med. 2011;365(12): $1118-1127$.

3. Zhong JH, Ke Y, Gong WF, et al. Hepatic resection associated with good survival for selected patients with intermediate and advanced-stage hepatocellular carcinoma. Ann Surg. 2014;260(2):329-340.

4. Dimitroulis D, Damaskos C, Valsami S, et al. From diagnosis to treatment of hepatocellular carcinoma: an epidemic problem for both developed and developing world. World J Gastroenterol. 2017;23(29):5282-5294.
5. Memczak S, Jens M, Elefsinioti A, et al. Circular RNAs are a large class of animal RNAs with regulatory potency. Nature. 2013;495(7441):333-338.

6. Jeck WR, Sorrentino JA, Wang K, et al. Circular RNAs are abundant, conserved, and associated with ALU repeats. RNA. 2013;19(2):141-157.

7. Guo JU, Agarwal V, Guo H, Bartel DP. Expanded identification and characterization of mammalian circular RNAs. Genome Biol. 2014;15(7):409.

8. Salzman J, Gawad C, Wang PL, Lacayo N, Brown PO. Circular RNAs are the predominant transcript isoform from hundreds of human genes in diverse cell types. PLoS One. 2012;7(2):e30733.

9. Li Z, Huang C, Bao C, et al. Exon-intron circular RNAs regulate transcription in the nucleus. Nat Struct Mol Biol. 2015;22(3):256-264.

10. Hansen TB, Jensen TI, Clausen BH, et al. Natural RNA circles function as efficient microRNA sponges. Nature. 2013;495(7441):384-388.

11. Meng S, Zhou H, Feng Z, et al. CircRNA: functions and properties of a novel potential biomarker for cancer. Mol Cancer. 2017;16(1):94.

12. Zhang J, Liu H, Hou L, et al. Circular RNA_LARP4 inhibits cell proliferation and invasion of gastric cancer by sponging miR-424-5p and regulating LATS1 expression. Mol Cancer. 2017;16(1):151.

13. Zhang Y, Liu H, Li W, et al. CircRNA_100269 is downregulated in gastric cancer and suppresses tumor cell growth by targeting miR-630. Aging. 2017;9(6):1585-1594. 
14. Liang G, Liu Z, Tan L, Su AN, Jiang WG, Gong C. HIF1alpha-associated circDENND4C promotes proliferation of breast cancer cells in hypoxic environment. Anticancer Res. 2017;37(8):4337-4343.

15. Kong Z, Wan X, Zhang Y, et al. Androgen-responsive circular RNA circSMARCA5 is up-regulated and promotes cell proliferation in prostate cancer. Biochem Biophys Res Commun. 2017;493(3):1217-1223.

16. Han D, Li J, Wang H, et al. Circular RNA MTO1 acts as the sponge of miR-9 to suppress hepatocellular carcinoma progression. Hepatology. 2017;66(4):1151-1164.

17. Li F, Zhang L, Li W, et al. Circular RNA ITCH has inhibitory effect on ESCC by suppressing the Wnt/beta-catenin pathway. Oncotarget. 2015;6(8):6001-6013.

18. Heldin CH, Moustakas A. Role of Smads in TGFbeta signaling. Cell Tissue Res. 2012;347(1):21-36.

19. Conn SJ, Pillman KA, Toubia J, et al. The RNA binding protein quaking regulates formation of circRNAs. Cell. 2015;160(6):1125-1134.

20. Liang D, Wilusz JE. Short intronic repeat sequences facilitate circular RNA production. Genes Dev. 2014;28(20):2233-2247.

21. Hatziapostolou M, Polytarchou C, Aggelidou E, et al. An HNF4alphamiRNA inflammatory feedback circuit regulates hepatocellular oncogenesis. Cell. 2011;147(6):1233-1247.

22. Jingushi K, Ueda Y, Kitae K, et al. miR-629 targets TRIM33 to promote TGFbeta/Smad signaling and metastatic phenotypes in ccRCC. Mol Cancer Res. 2015;13(3):565-574.
23. Chen J, Li Y, Zheng Q, et al. Circular RNA profile identifies circPVT1 as a proliferative factor and prognostic marker in gastric cancer. Cancer Lett. 2017;388:208-219.

24. Giannelli G, Koudelkova P, Dituri F, Mikulits W. Role of epithelial to mesenchymal transition in hepatocellular carcinoma. J Hepatol. 2016; 65(4):798-808.

25. Dupont S, Zacchigna L, Cordenonsi M, et al. Germ-layer specification and control of cell growth by Ectodermin, a Smad4 ubiquitin ligase. Cell. 2005;121(1):87-99.

26. He W, Dorn DC, Erdjument-Bromage H, Tempst P, Moore MA, Massague J. Hematopoiesis controlled by distinct TIF1 gamma and Smad4 branches of the TGFbeta pathway. Cell. 2006;125(5):929-941.

27. Dupont S, Mamidi A, Cordenonsi M, et al. FAM/USP9x, a deubiquitinating enzyme essential for TGFbeta signaling, controls Smad4 monoubiquitination. Cell. 2009;136(1):123-135.

28. Ding ZY, Jin GN, Wang W, et al. Reduced expression of transcriptional intermediary factor 1 gamma promotes metastasis and indicates poor prognosis of hepatocellular carcinoma. Hepatology. 2014;60(5): $1620-1636$. 


\section{Supplementary materials}

Table SI List of primers in this study

\begin{tabular}{ll}
\hline Primer name & Sequence $\left.\mathbf{( 5}^{\prime}-\mathbf{3}^{\prime}\right)$ \\
\hline circSMAD2-forward & ATGGACACAGGTTCGATACAAGAG \\
circSMAD2-reverse & CAACTGGCGGCGTGAATG \\
SMAD2 mRNA-forward & CTTTGTGCAGAGCCCCAATT \\
SMAD2 mRNA-reverse & CTTGTTACCGTCTGCCTTCG \\
GAPDH-forward & AGAAGGCTGGGGCTCATTTG \\
GAPDH-reverse & GCAGGAGGCATTGCTGATGAT \\
miR-629-forward & TGGGTTTACGTTGGGAGA \\
miR-629-reverse & GTGCAGGGTCCGAGGTATTC \\
U6-forward & CTCGCTTCGGCAGCACA \\
U6-reverse & AACGCTTCACGAATTTGCGT \\
\hline
\end{tabular}

Abbreviations: circSMAD2, circRNA SMAD2; GAPDH, glyceraldehyde 3-phosphate dehydrogenase; circRNA, circular RNA.

Table S2 Sequences of siRNA and miRNA mimics in this study

\begin{tabular}{lll}
\hline Gene name & Sense $\left(\mathbf{5}^{\prime}-\mathbf{3}^{\prime}\right)$ & Antisense $\left(\mathbf{5}^{\prime}-\mathbf{3}^{\prime}\right)$ \\
\hline si-circSMAD2 & AUGGACACAGGUUCGAUACTT & GUAUCGAACCUGUGUCCAUTT \\
miR-629 mimics & UGGGUUUACGUUGGGAGAACU & UUCUCCCAACGUAAACCCAUU \\
\hline
\end{tabular}

Abbreviations: circSMAD2, circRNA SMAD2; circRNA, circular RNA.

A

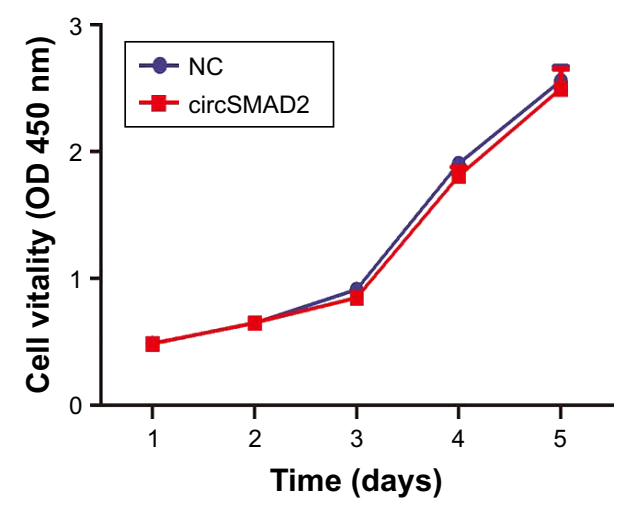

B
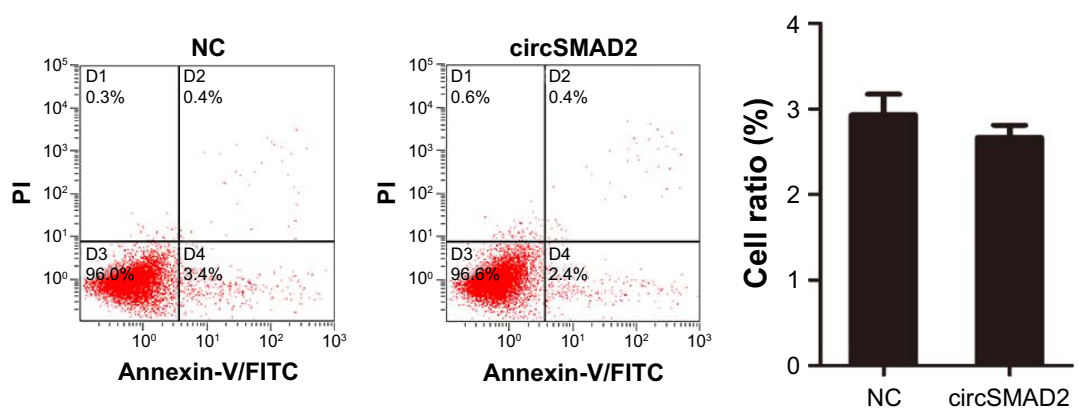

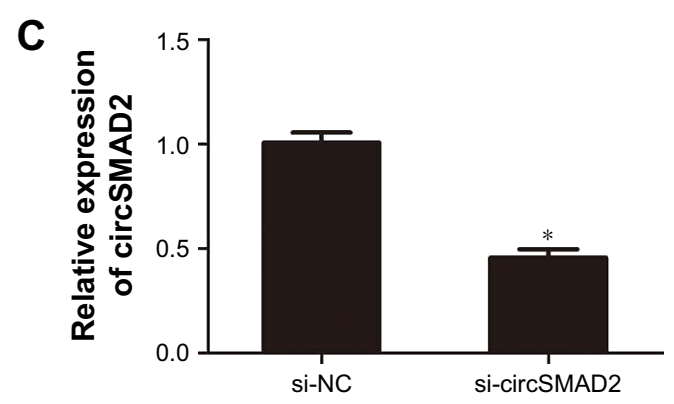

Figure SI Overexpressed circSMAD2 showed no significance on proliferation and apoptosis of HCC cells.

Notes: (A) CCK-8 assay showed no significant change in the proliferation of the circSMAD2-overexpressed HepG2 cells. (B) Flow cytometric assay showed no significant change in the apoptosis of the circSMAD2-overexpressed HepG2 cells. (C) Transfection of si-circSMAD2 significantly downregulated the expression of circSMAD2 in HCC cells. $* P<0.05$

Abbreviations: CCK-8, Cell Counting Kit-8; circSMAD2, circRNA SMAD2; HCC, hepatocellular carcinoma; circRNA, circular RNA; NC, negative control; OD, optical density; FITC, fluorescein isothiocyanate. 


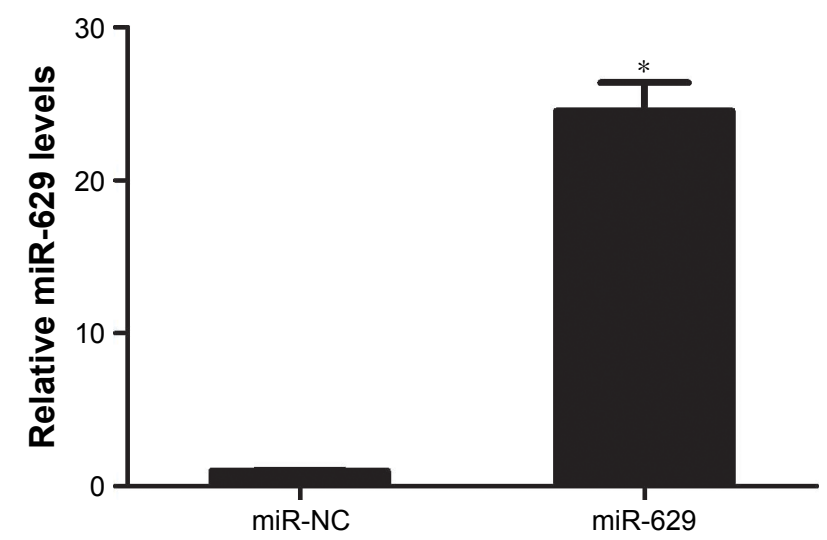

Figure S2 Transfection of miR-629 mimics significantly upregulated the expression of miR-629 in HepG2 cells. Note: $* P<0.05$

Abbreviation: NC, negative control.

\section{Publish your work in this journal}

OncoTargets and Therapy is an international, peer-reviewed, open access journal focusing on the pathological basis of all cancers, potential targets for therapy and treatment protocols employed to improve the management of cancer patients. The journal also focuses on the impact of management programs and new therapeutic agents and protocols on
Dovepress

patient perspectives such as quality of life, adherence and satisfaction. The manuscript management system is completely online and includes a very quick and fair peer-review system, which is all easy to use. Visit http://www.dovepress.com/testimonials.php to read real quotes from published authors.

Submit your manuscript here: http://www.dovepress.com/oncotargets-and-therapy-journal 